\title{
Renal Permeability Alteration Precedes Hypertension and Involves Bradykinin in the Spontaneously Hypertensive Rat
}

Gérard E. Plante, Martine Bissonnette, Martin G. Sirois, Domenico Regoli, and Pierre Sirois

Departments of Medicine and Pharmacology, University of Sherbrooke, Sherbrooke, Québec, Canada J1H 5 N4

\begin{abstract}
Vascular permeability disorders have been described in experimental models, as well as in human hypertension. We recently described the fact that vascular permeability to albumin is heterogeneous in the normal rat. In the present study, we examine the contents of Evans blue dye (EB) bound to albumin in selected organs of unanesthetized Wistar Kyoto (WKY) and in spontaneously hypertensive rats (SHR) at various stages of development of hypertension. EB was injected in the caudal vein of paired 4, 8, 12, and 16-wk-old WKY and SHR. Rats were killed 10 min after EB injection and extraction of the marker was measured in selected tissues. In additional 4 and 16-wk-old animals, bradykinin B1 and B2 receptor antagonists (BKA) were also injected with EB. Renal contents of EB bound to albumin were higher in the SHR than in the WKY: 196 \pm 9 , $202 \pm 10,182 \pm 7$, and $196 \pm 9$, compared with $158 \pm 8,155 \pm 7$, $138 \pm 7$, and $118 \pm 6 \mu \mathrm{g} / \mathrm{g}$ dry tissue, in the $4,8,12$, and 16-wkold rats, respectively. In the 4-wk-old SHR and WKY, blood pressure values were normal and comparable, yet the alteration in EB permeability was already present in the SHR. Both BKA failed to alter the renal EB extravasation in the WKY, but the B2-BKA restored the renal permeability to control levels in the SHR. We conclude that a selective defect in the renal vascular permeability to EB developed in the SHR. Since this finding precedes hypertension and is corrected by a selective B2-BKA, it is suggested that bradykinin is involved at an early stage of the disease in the SHR. (J. Clin. Invest. 1992. 89:2030-2032.) Key words: hypertension • vascular permeability • Evans blue • albumin $\bullet$ bradykinin antagonists
\end{abstract}

\section{Introduction}

The role of abnormal fluid balance has been repeatedly suggested in the etiology of hypertension, during the past decades (1-3). The distribution pattern of excess fluid between vascular and interstitial compartments in hypertensive patients remains controversial: a reduction (4-8), or no change in plasma volume $(9,10)$ were reported in such patients. Contradictory observations were also published regarding total extracellular vol-

Address correspondence to Dr. Gérard E. Plante, Department of Medicine, University of Sherbrooke, Sherbrooke, Quebec, Canada J1H 5 N4.

Received for publication 6 February 1992.

J. Clin. Invest.

(c) The American Society for Clinical Investigation, Inc.

$0021-9738 / 92 / 06 / 2030 / 03 \$ 2.00$

Volume 89, June 1992, 2030-2032 ume, which was either found increased $(11,12)$, or normal $(8$, $10)$ in this condition. More recently (13), selective augmentation of interstitial space was reported in 12-d-old spontaneously hypertensive rats (SHR). ${ }^{1}$ The mechanisms responsible for such abnormalities in body fluid compartments have not been elucidated, however. Transcapillary escape of albumin has been shown in hypertensive patients $(14,15)$, but in one such report (14), this abnormality was attributed to the nonspecific elevation of intraarterial pressure. Elevated plasma aldosterone and increased extracellular volume, which normalized by $25 \mathrm{~d}$ of age, was also reported in preweanling SHR (16). Because of the unique heterogeneous response of specific vascular beds to injection of vasoactive agonists (17), and because of potential disturbances in the permeability characteristics of these microcirculation beds in the pathophysiology of hypertension, we decided to examine the distribution of Evans blue bound to albumin (EB) in selected organs of SHR and WistarKyoto rats (WKY) of different ages $(4,8,12$, and 16 wk old). The results indicate, first, that selective renal sequestration of EB was obtained in the SHR, second, that the abnormality preceded the development of hypertension, third, that a specific antagonist of bradykinin B2 receptors restored this abnormality of vascular permeability. We conclude that an increased bradykinin-dependent renal permeability to EB exists in the SHR, and that such abnormality may contribute to the development of hypertension.

\section{Methods}

Six groups of SHR were compared to six paired WKY groups (each of six to eight animals). The first four paired groups included rats of different age and blood pressure values: $4,8,12$, and $16 \mathrm{wk}$. The two last pairs only included rats of 4 and 8 wk of age. All studies were conducted in awake animals, fasted the day before experiments, but allowed free water intake. Systolic blood pressure was measured by tail cuff plethysmography, two days before acute experiments. In all groups, a single injection of EB was performed in the caudal vein, and animals were killed by decapitation $10 \mathrm{~min}$ after the injections. In the last two groups, specific bradykinin (BK) B1 or B2 receptor antagonists ([Leu8]des-Arg9BK or D-Arg0[Hyp3,D-Phe7]BK, respectively) were injected individually $(25 \mu \mathrm{g} / \mathrm{kg})$ and simultaneously with EB. The specificity and inhibitory properties of these antagonists have been characterized previously in our laboratories $(18,19)$. After exsanguination, the following organs were dissected: skeletal muscle (left quadriceps), lungs, heart, liver, duodenum, pancreas, spleen, and kidney. The evaluation of wet and dry weights of each specimen, EB extraction and measurement were done according to methods described in previous

1. Abbreviations used in this paper: BK, bradykinin; EB, Evans blue; SHR, spontaneous hypertensive rats; WKY, Wistar Kyoto. 
papers $(17,20)$. EB contents of individual organs were expressed in micrograms per gram of dry tissue and taken as a measure of regional capillary permeability and interstitial sequestration of this marker. The results were averaged (six to eight measures) for each organ. Paired or unpaired comparison (Student's $t$ test), as indicated, was done between organs, age groups, as well as SHR and WKY strains.

\section{Results}

Fig. 1 illustrates the profile of systolic blood pressure in the two populations of rats examined at age $4,8,12$, and $16 \mathrm{wk}$. It is important to note that elevation of systolic pressure in the SHR was only apparent from week 8 . Therefore, SHR and WKY groups are comparable with respect to systolic pressure at week 4.

As shown in Table I, the pattern of EB distribution was markedly different from one selected organ to the other, in both SHR and WKY, and for all ages studied. EB accumulation was lowest in the pancreas, the lungs, and the skeletal muscle, whereas the kidneys and the spleen had more important accumulation of the marker. This pattern of EB distribution was generally comparable in both WKY and SHR groups, and remained as such from weeks 4 to 16 . However, there was a trend for EB bound to albumin sequestration in the kidney of WKY rats, to decrease between weeks 4 and 16, from $151 \pm 8$ to $116 \pm 11 \mu \mathrm{g} / \mathrm{g}$ dry tissue $(P<0.05)$. No such tendency was obtained for the kidney in the SHR group. Absolute EB accumulation in most tissues was not statistically different in WKY and SHR groups, except for the heart and kidney. Heart EB accumulation was greater (by $\sim 30 \%$ ) in SHR than in WKY rats at weeks 4 and $8(P<0.05)$. The difference in renal EB sequestration between SHR and WKY rats was more important: it averaged $31,63,51$, and $71 \mu \mathrm{g} / \mathrm{g}$ dry tissue, at weeks 4,8 , 12 , and 16 , respectively, all differences being statistically significant $(P<0.01)$. It is of interest, as illustrated in Fig. 2 , that EB accumulation was highest in SHR kidneys of all ages. More importantly, the phenomenon was already present in the 4-wkold SHR, at an age where the systolic blood pressure was comparable in both SHR and WKY rats, as shown in Fig. 1.

The effect of B1 and B2 bradykinin receptor antagonists on the kidney EB distribution is illustrated in Fig. 3. In 4 and 8-wk-old animals, renal EB was again higher $(P<0.01)$ in the SHR than in the WKY $(198 \pm 8$ and $188 \pm 6$, compared to

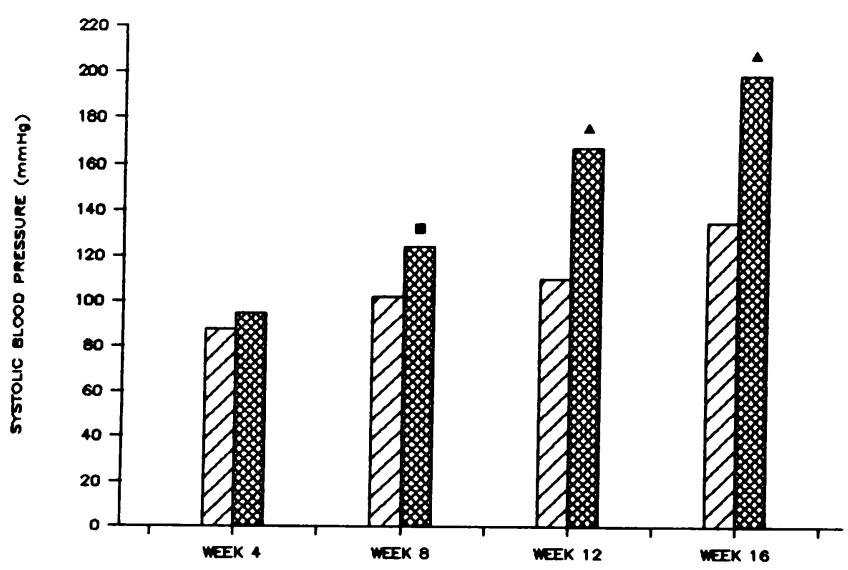

Figure 1. Systolic blood pressure in WKY and SHR groups at 4, 8, 12, and 16 weeks of age. Statistical significance for observed differences between groups is indicated by squares $(P<0.05)$ and triangles $(P<0.01)$. . W, WKY; $\square$, SHR.
Table I. Organ Distribution of Evans Blue ( $\mu g / g$ Dry Tissue) Measured at $10 \mathrm{Min}$

\begin{tabular}{lcccc}
\hline & \multicolumn{4}{c}{ Control animals (WKY) } \\
\cline { 2 - 5 } & Week 4 & Week 8 & Week 12 & Week 16 \\
\hline Pancreas & $44 \pm 6$ & $51 \pm 6$ & $81 \pm 15$ & $64 \pm 6$ \\
Lung & $51 \pm 5$ & $42 \pm 9$ & $60 \pm 17$ & $79 \pm 15$ \\
Muscle & $59 \pm 8$ & $58 \pm 16$ & $79 \pm 6$ & $51 \pm 19$ \\
Duodenum & $84 \pm 6$ & $95 \pm 14$ & $118 \pm 14$ & $88 \pm 12$ \\
Heart & $93 \pm 14$ & $75 \pm 4$ & $132 \pm 19$ & $88 \pm 18$ \\
Liver & $98 \pm 15$ & $96 \pm 8$ & $124 \pm 10$ & $103 \pm 16$ \\
Kidney & $151 \pm 8$ & $125 \pm 11$ & $133 \pm 12$ & $116 \pm 11$ \\
Spleen & $172 \pm 23$ & $167 \pm 13$ & $186 \pm 19$ & $178 \pm 16$ \\
& & Hypertensive animals (SHR) & \\
\cline { 2 - 5 } & & & & \\
Pancreas & $69 \pm 7$ & $59 \pm 8$ & $93 \pm 16$ & $57 \pm 10$ \\
Lung & $76 \pm 5$ & $40 \pm 9$ & $83 \pm 13$ & $85 \pm 4$ \\
Muscle & $75 \pm 8$ & $76 \pm 16$ & $98 \pm 22$ & $90 \pm 30$ \\
Duodenum & $106 \pm 15$ & $86 \pm 6$ & $108 \pm 12$ & $99 \pm 15$ \\
Heart & $130 \pm 8^{*}$ & $101 \pm 11^{*}$ & $127 \pm 27$ & $117 \pm 20$ \\
Liver & $143 \pm 17$ & $110 \pm 10$ & $122 \pm 7$. & $108 \pm 18$ \\
Kidney & $182 \pm 4^{\ddagger}$ & $188 \pm 7^{\ddagger}$ & $204 \pm 16^{\ddagger}$ & $187 \pm 8^{\ddagger}$ \\
Spleen & $194 \pm 21$ & $193 \pm 15$ & $212 \pm 26$ & $184 \pm 20$ \\
& & & & \\
\hline
\end{tabular}

Statistical significance (SHR-WKY): ${ }^{*} P<0.05,{ }^{\ddagger} P<0.01$.

$160 \pm 9$ and $142 \pm 7 \mu \mathrm{g} / \mathrm{g}$ dry tissue, respectively). Neither B1 and B2 bradykinin receptor antagonists per se altered renal EB distribution in WKY. There was also no effect of B1 bradykinin receptor antagonist on EB distribution in the SHR. But the B2 bradykinin antagonist restored the renal EB accumulation, in both 4 and 8-wk-old SHR, to $162 \pm 7$ and $144 \pm 6 \mu \mathrm{g} / \mathrm{g}$ dry tissue, respectively, values not different from control data obtained in the WKY.

\section{Discussion}

The observations described in this paper represent new information related to the pathophysiology of hypertension in the SHR: first, it demonstrates clearly a selective defect in vascular permeability to EB in the SHR strain; second, the selective renal sequestration of EB is apparent before the development

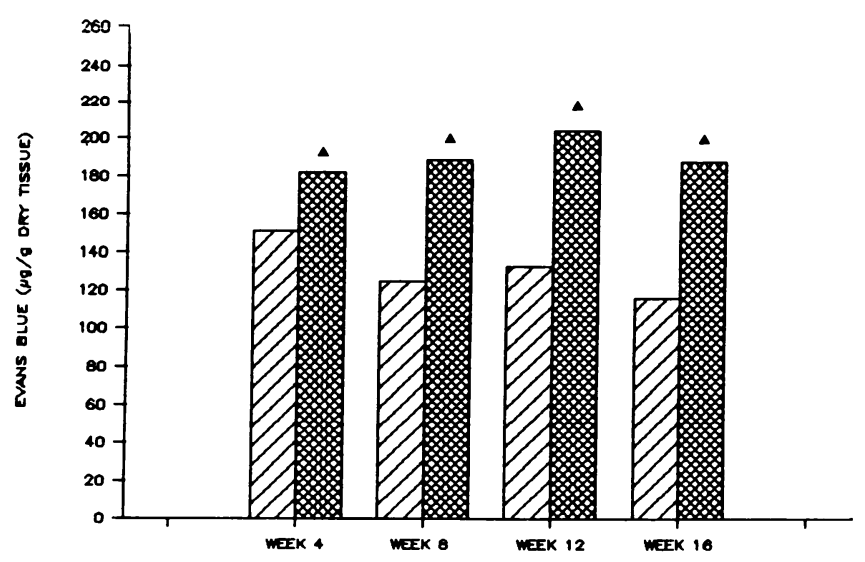

Figure 2. Kidney contents of Evans blue dye in WKY and SHR groups at $4,8,12$, and 16 weeks of age. Statistical significance as in Fig. 1. . WKY; $\mathbf{n}$, SHR. 


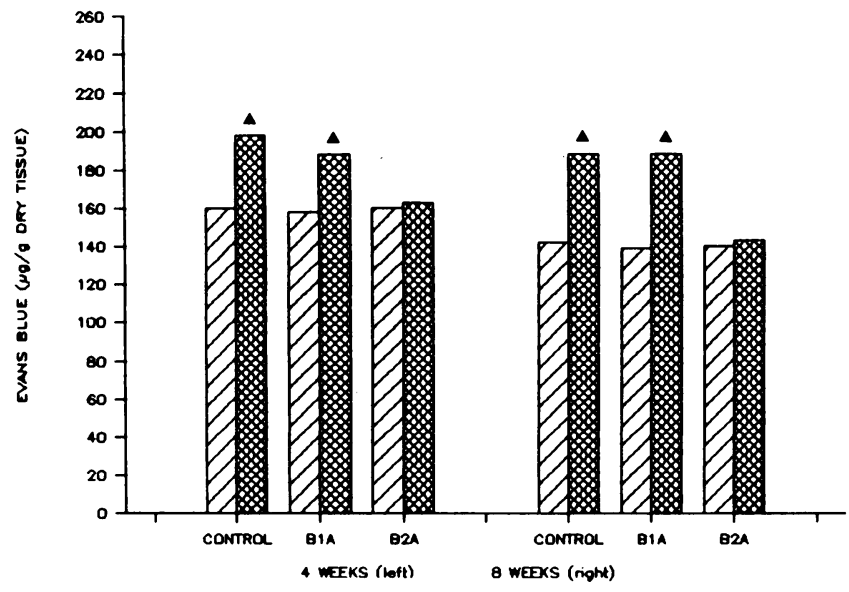

Figure 3. Effect of $\mathrm{B} 1$ and $\mathrm{B} 2$ bradykinin antagonists $(B 1 A$ and $B 2 A)$ on kidney Evans blue extravasation in WKY and SHR at 4 (left) and 8 (right) weeks of age. Statistical significance as in Fig. 1. ש, WKY; 圆, SHR.

of hypertension; third, B2 bradykinin receptors appear to play a critical role, in mediating this unique permeability abnormality. Such findings, to our knowledge, have not been reported in this hypertensive animal model, nor in human arterial hypertension.

The method used in this study to demonstrate eventual permeability disorders in SHR, evaluates the leak of albumin from vascular volume to interstitium, since Evans blue is mainly bound to this plasma protein. Validation of this approach to examine regional capillary endothelial permeability has been published in previous papers from this $(17,20)$ and other laboratories $(21,22)$.

Why did EB preferentially accumulate in the SHR kidney? Compared with most major organs, the kidney has the highest blood flow per gram of tissue (23). In addition, the renal microcirculation possesses a high permeability coefficient (24). However, it is unlikely that the kidney EB sequestration observed in SHR was merely due to these properties of the renal microcirculation. In fact, the permeability features of hepatic, splenic, and gastrointestinal submucosal microcirculation are quite comparable, yet accumulation of EB in these organs was not different in SHR and WKY groups.

Why did EB accumulate in the 4-wk-old SHR kidney? The higher renal microcirculation permeability to serum albumin found in SHR compared with the WKY could not reasonably be related to high intraarterial pressure, as suggested for whole body albumin extravasation in human hypertension (14). Since 4-wk-old animals examined in the present study were normotensive, it then becomes critical to look at eventual primary abnormalities (physical and/or humoral) of the renal microcirculation in the SHR, to explain the selective and early renal EB sequestration documented in the present study. It is reasonable to propose that the renal bradykinin system, in particular the B2 receptors, initiates the selective vascular permeability changes leading to EB accumulation in the renal interstitium, weeks before arterial hypertension develops.

\section{Acknowledgments}

We recognize the expert technical assistance of Angèle Chainey.

This research was supported in part by Le Fonds de la Recherche en Santé du Québec (FRSQ 568-I).

\section{References}

1. Lucas, L., and M. A. Floyer. 1974. Changes in body fluid distribution and interstitial tissue compliance during the development and reversal of experimental renal hypertension in the rat. Clin. Sci. Mol. Med. 47:1-11.

2. Tarazi, R. C. 1976. Hemodynamic role of extracellular fluid in hypertension. Circ. Res. 38(Suppl. II):II-73-II-83.

3. Wenting, G. J., A. J. Man In't Veld, R. P. Nerhoevan, F. H. M. Derkx, and M. A. D. H. Schalekamp. 1977. Volume-pressure relationships during development of mineralocorticoid hypertension in man. Circ. Res. 40(Suppl. I):I-163-I170.

4. Hansen, J. 1968. Blood volume exchangeable sodium in essential hypertension. Acta Med. Scand. 184:517-523.

5. Rochlin, D. B., T. Shohl, and W. S. Blakemore. 1960. Blood volume changes associated with essential hypertension. Surg. Gynecol. \& Obstet. 111:569-575.

6. Tarazi, R. C., H. P. Dustan, and E. D. Frohlich. 1969. Relation of plasma to interstitial fluid volume in essential hypertension. Circulation. 40:357-365.

7. Tarazi, R. C., E. D. Frohlich, and H. P. Dustan. 1968. Plasma volume in men with essential hypertension. N. Engl. J. Med. 278:762-765.

8. Tibblin, G., S. E. Bergentz, J. Bjure, and L. Wilhelmsen. 1966. Hematocrit, plasma protein, plasma volume, and viscosity in early hypertensive disease. $\mathrm{Am}$. Heart J. 72:165-171.

9. Cranston, W. I., and W. Brown. 1963. Diurnal variation in plasma volume in normal and hypertensive subjects. Clin. Sci. (Lond.). 25:107-112.

10. Walser, M., B. J. Duffy, and H. W. Griffith. 1956. Body fluids in hypertension and mild heart failure. JAMA (J. Am. Med. Assoc.). 160:858-864.

11. Ross, E. J. 1956. Total exchangeable sodium in hypertensive patients Clin. Sci. (Lond.). 15:81-88.

12. Teng, H. C., A. P. Shapiro, and A. Grollman. 1954. Volume of the fluid compartments in human and experimental hypertension. Metabolism Clin. \& Exp. 3:405-409.

13. Mullins, M. M. Body fluid volumes in prehypertensive spontaneously hypertensive rats. 1983. Am. J. Physiol. 244:H652-H655.

14. Parving, H. H and F. Gyntelberg. 1973. Transcapillary escape rate of albumin and plasma volume in essential hypertension. Circ. Res. 32:643-651.

15. Ulrych, M. 1976. The role of vascular capacitance in the genesis of essential hypertension. Clin. Sci. Mol. Med. 51:203s-205s.

16. Mullins, M. M. L. I. Kleinman, P. T. Russell, and L. S. Srivastava. 1982 Plasma aldosterone concentrations in neonatal spontaneously hypertensive rats. Life Sci. 31:2751-2755.

17. Sirois, M. G., S. Jancar, P. Braquet, G. E. Plante, and P. Sirois. 1988. PAF increases vascular permeability in selected tissues: effect of BN-52021 and L-655,240. Prostaglandins. 36:631-644.

18. Rhaleb, N. E., S. Dion, J. Barabe, N. Roussi, D. Jukic, G. Drapeau, and D. Regoli. 1989. Receptors for kinins in isolated arterial vessels of dogs. Eur. J. Pharmacol. 162:419-427.

19. Lortie, M., D. Regoli, N. E. Rhaleb, and G. E. Plante. 1992. Dissociation of hemodynamic and tubular effects of intrarenal bradykinin infusion. Am. J. Physiol. 262:R72-R76.

20. Sirois, M. G., G. E. Plante, P. Braquet, and P. Sirois. 1990. Role of eicosanoids in PAF-induced increases of the vascular permeability in rat airways. Br. J. Pharmacol. 101:896-900.

21. Leveen, H. H., and W. H. Fishman. 1947. Combination of Evans blue with plasma protein: its significance in capillary permeability studies, blood dye disappearance curves, and its use as a protein tag. Am. J. Physiol. 151:26-33.

22. Rogers, D. F., P. Boschetto, and P. J. Barnes. 1989. Correlation between Evans blue dye and radiolabeled albumin in guinea pig airways in vivo. J. Pharmacol. Methods. 21:309-315.

23. Barger, A. C., and J. A. Herd. 1971. The renal circulation. N. Engl. J. Med. 284:482-490.

24. Venkatachalam, M. A., and M. J. Karnovsky. 1972. Extravascular protein in the kidney: an ultrastructural study of its relation to renal peritubular capillary permeability using protein tracers. Lab. Invest. 27:435-442. 